This item was submitted to Loughborough's Research Repository by the author.

Items in Figshare are protected by copyright, with all rights reserved, unless otherwise indicated.

\title{
Biocompatibility and immunogenicity of decellularised allogeneic aorta in the orthotopic rat model
}

\section{PLEASE CITE THE PUBLISHED VERSION}

https://doi.org/10.1089/ten.tea.2018.0037

\section{PUBLISHER}

(c) Mary Ann Liebert, Inc.

\section{VERSION}

AM (Accepted Manuscript)

\section{PUBLISHER STATEMENT}

Final publication is available from Mary Ann Liebert, Inc., publishers https://doi.org/10.1089/ten.tea.2018.0037.

\section{LICENCE}

CC BY-NC-ND 4.0

\section{REPOSITORY RECORD}

Katsimpoulas, Michalis, Lucrezia Morticelli, loanna Gontika, Artemis Kouvaka, Panagiotis Mallis, Daniele Dipresa, Ulrike Boer, et al.. 2019. "Biocompatibility and Immunogenicity of Decellularised Allogeneic Aorta in the Orthotopic Rat Model”. figshare. https://hdl.handle.net/2134/37174. 
Biocompatibility and Immunogenicity of Decellularised Allogeneic Aorta in the Orthotopic Rat Model

2 Michalis Katsimpoulas $1,2^{*}$, Lucrezia Morticelli3*, Ioanna Gontika4, Artemis Kouvaka3, Panagiotis Mallis4, Daniele

3 Dipresa3, Ulrike Böer3, Bisharah Soudah5, Axel Haverich3,6, Efstathios Michalopoulos4, Sotiris Korossis3,6,7

1. Centre of Clinical, Experimental Surgery and Translational Research, Biomedical Research Foundation of the Academy of Athens, 4 Soranou Ephesius Street, 11527 Athens, Greece;

7 2. Attikon Animal Hospital, Lavriou Av., 19002 Paiania, Greece;

8 3. Lower Saxony Centre for Biomedical Engineering Implant Research and Development, Hannover Medical School, Stadtfelddamm 34, 30625 Hannover, Germany;

4. Hellenic Cord Blood Bank, Biomedical Research Foundation Academy of Athens, 4 Soranou Ephesius Street, 11527 Athens, Greece;

5. Institute for Pathology, Hannover Medical School, Carl-Neuberg-Str. 1, 30625 Hannover, Germany;

6. Department of Cardiothoracic, Transplantation and Vascular Surgery, Hannover Medical School, Carl-Neuberg-Str. 1, 30625 Hannover, Germany;

7. Centre for Biological Engineering, Wolfson School of Mechanical, Electrical and Manufacturing Engineering, Loughborough University, Asby Road, LE11 3TU Loughborough, United Kingdom.

* Contributed equally to this work as first authors

\section{Corresponding Author}

Michalis Katsimpoulas, DVM, SRS, PhD

Center of Clinical, Experimental Surgery and Translational Research, Biomedical Research Foundation of the Academy of Athens, 4 Soranou Ephesius Street, 11527 Athens, Greece

T: +302106597367

F: +302106597365

E-mail: mkatsiboulas@bioacademy.gr

Lucrezia Morticelli, PhD

Lower Saxony Centre for Biomedical Engineering Implant Research and Development, Department of Cardiothoracic,

30 Transplantation and Vascular Surgery, Hannover Medical School, Stadtfelddamm 34, 30625 Hannover, Germany

$31 \quad \mathrm{~T}:+495115328422$

$32 \quad F:+495115328314$

Email: morticelli.lucrezia@mh-hannover.de

Ioanna Gontika, MSc 
F: +302106597342

40

E-mail: igontika@bioacademy.gr

41

Artemis Kouvaka, PhD

Lower Saxony Centre for Biomedical Engineering Implant Research and Development, Department of Cardiothoracic,

Transplantation and Vascular Surgery, Hannover Medical School, Stadtfelddamm 34, 30625 Hannover, Germany

45

T: +495115327797

46

F: +495115328314

47

Email: kouvaka.artemis@mh-hannover.de

48

49

Panagiotis Mallis, MSc

50

Hellenic Cord Blood Bank, Biomedical Research Foundation of the Academy of Athens, 4 Soranou Ephesius Street, 51 11527 Athens, Greece

52

$\mathrm{T}:+302106597331$

53

F: +302106597342

54

E-mail: pmallis@bioacademy.gr

55

Daniele Dipresa, PhD

Lower Saxony Centre for Biomedical Engineering Implant Research and Development, Department of Cardiothoracic,

Transplantation and Vascular Surgery, Hannover Medical School, Stadtfelddamm 34, 30625 Hannover, Germany

T: +49 5115327797

60

F: +495115328314

61

Email: dipresa.daniele@mh-hannover.de

62

Ulrike Böer, PhD

64

Lower Saxony Centre for Biomedical Engineering Implant Research and Development, Department of Cardiothoracic,

$\mathrm{T}:+495115321453$

F: +495115328314

68

Email: boeer.ulrike@mh-hannover.de

69

Bisharah Soudah, PhD

T: +49 5115324512

F: +495115325799

Email: soudah.bisharah@mh-hannover.de

Axel Haverich, Dr.h.c. 
Department of Cardiothoracic, Transplantation and Vascular Surgery, Hannover Medical School, Carl-Neuberg-Str. 1, 30625 Hannover, Germany

T: +495115326580

F: +495115325404

Email: haverich.axel@mh-hannover.de

\section{Efstathios Michalopoulos, PhD}

Hellenic Cord Blood Bank, Biomedical Research Foundation of the Academy of Athens, 4 Soranou Ephesius Street, 11527 Athens, Greece

T: +302106597331

F: +302106597342

E-mail: smichal@bioacademy.gr

\section{Sotirios Korossis, $\mathrm{PhD}$}

Centre for Biological Engineering, Wolfson School of Mechanical, Electrical and Manufacturing Engineering, Loughborough University, Asby Road, LE11 3TU Loughborough, United Kingdom.

T: +44 1509227651

Email: s.korossis@lboro.ac.uk

\section{Introduction}

Peripheral arterial disease is a highly prevalent and debilitating condition which affects more than 25 million patients in Europe and North America alone (Criqui et al., 1985; Fowkes et al., 1991). Peripheral arterial disease leads to the damage of blood vessels, which can be replaced by synthetic, autologous or allogeneic vascular grafts. Current replacement approaches employ biocompatible materials solutions that are not able to regenerate or grow with the patient. The gold standard for vascular grafting is autologous tissue, such as reversed saphenous vein graft (SVG) (Koyama et al., 2014). In despite of its low-cost and non-immunogenicity, autologous tissue does not represent the ideal solution due to its limited availability and size mismatch (Kakisis et al., 2005). Synthetic grafts, made of expandedpolytetrafluoroethylene (ePTFE) or Dacron, have been reported to give successful outcomes in large diameter arterial reconstructions $(>8 \mathrm{~mm})$, but not for small diameter arterial reconstructions $(<5 \mathrm{~mm})$, due to thrombosis and limited reendothelialization (Whittermore et al., 1989; Yu et al., 2013). In addition, cryopreserved arterial allogeneic grafts have a limited availability and durability, due to calcification, immunogenicity and thrombogenicity (Daping et al., 2009). The shortcomings of the conventional grafts have prompt the investigation of new approaches for peripheral artery reconstructions, such as the fabrication of tissue-engineered small-diameter vessels. Several studies have reported on the development of small-diameter vascular using naturally derived proteins, such as collagen and fibrin (Clark et al., 
1995 and Swartz et al., 2005, Dietrich et al., 2012, Aper et al., 2016, Schneider-Bartholda et al., 2016), and synthetic polymers (Roh et al., 2010). However, the inadequate mechanical properties of these grafts (Chaouat et al., 2006; Zhang et al., 2007; Zorlutuna et al., 2009) have prompted workers in the field to adopt alternative approaches, such as the use of decellularized tissue, which has been successfully used for tissue reconstructions, such as heart valves, bladder, tendons and meniscus (Affonso da Costa et al., 2005; Atala et al., 2006; Ingram et al., 2007; Bobylev et al., 2014; Neumann et al., 2014; Luo et al., 2014; Sarikouch et al., 2016, ). Decellularization represents a promising approach for overcoming the limited availability of autologous small-diameter vascular conduits, as well as the limited availability of histocompatible allogeneic grafts since decellularisation has the potential to remove the major antigenic determinants of allogeneic grafts. The latter has been effectively demonstrated by the successful translation of decellularised aortic and pulmonary allogeneic heart valves in the clinical setting (Sarikouch et al., 2016; Neumann et al., 2014), whereas a number of studies have reported promising results with decellularized small-diameter vascular grafts (Gui et al., 2009, 2010; Hwang et al., 2011; Dall'Olmo et al., 2014;). The present study was a part of an overarching project that was aimed at creating a small-caliber arterial graft for clinical use, utilizing the decellularised rat aorta. This approach was chosen due to the arterial properties of the rodent graft and its suitable size for small-caliber arterial reconstructions in humans. To the best of the authors' knowledge, such an approach has not been reported in the literature. Dark Agouti (DA) rat abdominal aortas were decellularized using the widely employed detergent sodiumdodexyl-sulfate (SDS) (Hashimoto et al., 2010; Santoso et al., 2014; Struecker et al., 2014; Paniagua et al., 2015) in 3[(3-cholamidopropyl) dimethylammonio]-1-propanesulfonate (CHAPS) buffer (Gui et al., 2009, 2010; Hwang et al., 2011; Dall'Olmo et al., 2014). Previously, we have reported on the effect of this modified decellularization protocol on the mechanical and histological integrity of DA rat aortas in vitro (Katsimpoulas et al., 2015). The present study was focused on assessing the performance of the decellularised graft under orthotopic allogeneic transplantation in inbred Wistar (W) rats without immunosuppression, prior to proceeding to heterotopic xenotransplantation. This specific animal model was chosen since the inbred W and inbred DA rats differed in both their major and minor histocompatibility loci (MHC I and II). Moreover, the orthotopic transplantation was chosen in order to assess the decellularised graft in the more demanding, higher pressure environment of the aorta. The elicited immune response was evaluated in terms of inflammatory cell infiltration into the implanted grafts, as well as in terms of the histoarchitectural and biomechanical changes in the implanted grafts, after 6 weeks of implantation.

\section{Material and Methods}

\section{Experimental animals}


The study was conducted using 40 male inbred $W$ and 20 male inbred DA rats, which were maintained in accordance with the European Directive 2010/63 for the protection of animals used for scientific purposes and the "Guide for the Care and Use of Laboratory Animals" (Guillen, 2012). All procedures were carried out in the animal facility of the Biomedical Research Foundation of the Academy of Athens (EL25BIO003). The experimental protocol was approved by the competent veterinary authority of the Biomedical Research Foundation of the Academy of Athens (7047/27-112012), in accordance with the Greek legislation on the protection of experimental animals and the European Directive 2010/63.

\section{Specimen procurement and dissection}

The abdominal aorta was approached via a midline laparotomy incision under isoflurane anaesthesia (IsoFlo, Abbott, UK; $2.5 \%, 0.5 \mathrm{l} / \mathrm{min}$ 02), as described by Onuta et al. (2007) and Hwang et al. (2011), and the side branches were carefully cauterized (HTC, FLAB, Italy). The aorta was then mobilized and transected between a point distally to the left renal artery and proximally to the iliac bifurcation. The harvested conduit was flushed with normal saline (Onuta et al., 2007). Twenty native aortas from 10 DA (DA-W group; allogeneic control) and $10 \mathrm{~W}$ (W-W group; syngeneic control) rats were orthotopically implanted untreated in W rats. Moreover, $10 \mathrm{DA}$ aortas were decellularized using a modified, protocol (Katsimpoulas et al. 2015) and implanted orthotopically in W rats ( $n=10$; decell DA-W group). The implanted aortas were explanted after 6 weeks and tested histologically ( $n=2$ from each group), immunohistochemically ( $n=2$ from each group) and biomechanically ( $\mathrm{n}=6$ from each group).

\section{Decellularization}

The decellularisation of the rodent abdominal aortas was carried out as described previously (Gui et al., 2009) with modifications (Katsimpoulas et al. 2015). Briefly, 20 aortas of approximately $15 \mathrm{~mm}$ in length were incubated with CHAPS solution (8 mmol/L CHAPS [APPLICHEM, US], $1 \mathrm{~mol} / \mathrm{L} \mathrm{NaCl}$, and $25 \mathrm{mmol} / \mathrm{L}$ EDTA in phosphate buffer saline [PBS; Sigma, Germany]) at pH 8 for $22 \mathrm{~h}$, followed by brief washes in PBS. The aortas were further incubated with SDS solution (1.8mmol/L SDS [Sigma, Germany], $1 \mathrm{~mol} / \mathrm{L} \mathrm{NaCl}$, and $25 \mathrm{mmol} / \mathrm{L}$ EDTA in PBS) at pH7.5 for $24 \mathrm{~h}$, followed by 3 washes, 5 min each, in PBS to completely remove the detergent. Finally, and modifying the previously described protocol (Gui et al., 2010), the aortas were incubated at $37^{\circ} \mathrm{C}$ for $48 \mathrm{~h}$ in alpha minimal essential medium (a-MEM, Gibco Life Technology, Germany), containing 40\% (v/v) fetal bovine serum (FBS, Gibco Life Technology, Germany) and 1000 U/mL penicillin-streptomycin (Gibco Life Technology, Germany). FBS was added for removal of residual DNA from the scaffolds (Gui L et al. 2010), since it contains DNAses and RNAses activated at $37^{\circ} \mathrm{C}$ in incubator. In addition, FBS can 
be stored for a minimum 10 days at $4^{\circ} \mathrm{C}$ without altering its properties, while it has been approved by FDA for several cellular and tissue engineered products. On the other hand, protocols that use external DNAses and RNAses or EGM-2 is of a higher cost due to the fact that a nuclease solution must be used immediately and is stable only for $3-5 \mathrm{~h}$, creating storage issues (Ingram, J.H., et al. 2007; Gui et al., 2009). Moreover, this step does not alter the mechanical properties of the scaffold, as assessed by our group (Katsimpoulas et al. 2015). All decellularisation steps were carried out under agitation and sterile conditions.

\section{Implantation procedure}

Anesthesia and aortic mobilization were performed as in the case of donor preparation while heparin was added in a dose of $10 \mathrm{IU} / 100 \mathrm{~g}$ body weight intravenously. The aorta was transplanted just distally to the renal artery and proximally to the iliac bifurcation in an end-to-end continuous fashion with 8-0 polypropylene suture (Prolene, Ethicon, USA). No

\section{Histology} additional anticoagulation was administered postoperatively. Postoperatively, $5 \mathrm{mg} / \mathrm{kg} \mathrm{SC}$ Carprofen (Rimadyl, Pfizer, UK) daily and $150 \mathrm{mg} / \mathrm{kg} \mathrm{SC} \mathrm{Amoxicillin} \mathrm{(Amoxil,} \mathrm{GlaxoSmithKline,} \mathrm{UK)} \mathrm{twice} \mathrm{a} \mathrm{day} \mathrm{was} \mathrm{administered} \mathrm{for} 3$ days, for pain and antibioprophylaxis, respectively. The recipient $\mathrm{W}$ rats were euthanized 6 weeks postoperatively, and the grafts, with $\sim 2 \mathrm{~cm}$ surrounding tissue, were removed from the recipients.

The tissue morphology and cellular content of the native, decellularised and explanted grafts was assessed by histology. Whole aorta segments measuring $10 \mathrm{~mm}$ in length were isolated and fixed in 10\% (v/v) neutral buffered formalin (SigmaAldrich) overnight at $4^{\circ} \mathrm{C}$. The samples were then dehydrated by sequential immersion in graded concentrations of ethanol $(70 \% \mathrm{v} / \mathrm{v}, 90 \% \mathrm{v} / \mathrm{v}$ and $100 \% \mathrm{v} / \mathrm{v})$ in distilled water for $1 \mathrm{~h}$ each, before they were immersed three times in xylene for $1 \mathrm{~h}$ each time. The samples were then placed into metal molds that were partially filled with paraffin. The molds were transferred onto a cold plate, to initiate wax solidification and secure sample orientation, and then were filled with paraffin. The molds were placed onto the cold plate again, until the wax was completely solid. Subsequently, the paraffin blocks were removed from the molds and sectioned using a microtome at a thickness of $6 \mu \mathrm{m}$. Circumferential sections were cut from the central region of the grafts, in order to avoid the suture lines. Subsequently, the sections were transferred into a water bath at $50^{\circ} \mathrm{C}$ and onto microscope slides. The slides with the tissue sections were dried on a $60^{\circ} \mathrm{C}$ hotplate. Prior to staining, the sections were dewaxed by sequential immersion in 2 individual pots with xylene for 10 min each, and were then sequentially rehydrated by immersion in graded concentrations of ethanol $(2 \times 5$ min $100 \%$ $\mathrm{v} / \mathrm{v}, 1 \times 2 \mathrm{~min} 95 \% \mathrm{v} / \mathrm{v}, 1 \times 2 \mathrm{~min} 70 \% \mathrm{v} / \mathrm{v}$ ) in distilled water. The general histoarchitecture of the grafts was evaluated using 
standard haematoxylin and eosin (H\&E; Merck) staining. Sections were immersed into Mayer's haematoxylin for 1 min, rinsed under tap water for $5 \mathrm{~min}$ and then immersed into eosin for $3 \mathrm{~min}$. Masson's trichrome was used to visualize collagen fibre alignment and cell nuclei according to the method described by the manufacturer (Sigma-Aldrich). Briefly, sections were treated with Weigert's iron haematoxylin for $5 \mathrm{~min}$, then in Biebrich scarlet-acid fuchsin and aniline blue for 6 and $5 \mathrm{~min}$, respectively. Elastica Van Gieson was used to visualise elastic fibres and cell nuclei according to the manufacturer's instructions (Sigma Aldrich). Briefly, sections were immersed in Weigert's resorcin fuchsin solution for 11 min, followed by Weigert's iron haematoxylin and picrofuchsin solution for 5 and $2 \mathrm{~min}$, respectively. All stained sections were dehydrated again in graded concentrations of ethanol $(70 \% \mathrm{v} / \mathrm{v}, 90 \% \mathrm{v} / \mathrm{v}$ and $100 \% \mathrm{v} / \mathrm{v})$ in distilled water, cleared with xylene and mounted with Corbit Balsam mountant. Images were captured using a Nikon TE300 Eclipse light microscope, incorporating a Nikon Digital Sight DS-U3 camera controller, and processed through the NIS-Elements D Microscope Imaging Software (Nikon Instruments).

\section{Immunohistochemistry}

The elicited immune response was evaluated in terms of inflammatory cell (T-cells, monocytes and macrophages) infiltration by immunohistochemical staining. The presence of smooth muscle cells (SMCs)/fibroblasts and endothelial cells (ECs) in the native and explanted grafts was also investigated by immunohistochemical staining. Whole aorta samples measuring $10 \mathrm{~mm}$ in length, were isolated and fixed in zinc fixative at $4^{\circ} \mathrm{C}$, dehydrated, and embedded in paraffin wax, as described above. Circumferential sections $(6 \mu \mathrm{m})$ were cut close to the central region of the samples, dewaxed, and stained. Monoclonal antibodies against CD31 (ECs; PECAM-1(M-20)-R; Santa Cruz Biotech), calponin (SMCs/myofibroblasts; 46794, Abcam), CD68 (monocytes and circulating and tissue macrophages; MCA341GA; AbD Serotec), CCR7 (M1 macrophage sub-type; rabbit monoclonal Anti-CCR7 antibody [Y59], ab32527, Abcam) and CD206 (M2 macrophage sub-type; rabbit polyclonal to Mannose Receptor, ab64693, Abcam) were used to stain sections of native and explanted aortas (Brown et al.,2009; Brown et al., 2012). Explanted aortas were also stained with anti-CD3

218 (T-cells at all stages of development; ab5690; Abcam) and anti-CD4 (T-regulatory and T-helper cells; ab125711; Abcam) 219 antibodies. Fresh porcine mitral valve posterior leaflet was used as positive control for calponin staining, whereas spleen was used as positive control for T-cell staining. Isotype control antibodies (normal rabbit IgG for CD31, calponin and CD3, Calbiochem; mouse lgG1 for CD4 and CD68, Dako) and omission of the primary antibody (secondary antibody control) served as negative controls. Immunolabelling of anti-CD31, anti-CD68, anti-CD3 and anti-CD4 was carried out using the EnVision®+ Dual Link System-HRP (DAB+) (K4065, Dako). Hydrogen peroxide (Sigma) was used to block endogenous peroxidase. Images were captured under light microscopy, as described above. Immunofluorescence 
staining was conducted for the immunolabelling of anti-calponin, anti-CCR7 and anti-CD206. Briefly, samples were incubated in blocking medium (1\% w/v BSA in PBS) for 60 min at RT, before they were washed once in TBS/Tween and twice in TBS, for 5 min each time. Incubation in primary antibody was carried out for $1 \mathrm{~h}$ at RT. All samples were then washed twice in TBS/Tween and twice in TBS for 5 min each and incubated with the secondary antibody (Alexa Fluor 488, donkey anti-rabbit, lgG, Jackson) for $30 \mathrm{~min}$ in the dark at RT. The samples were then washed shortly in TBS/Tween and TBS and incubated in RotiBMount FluorCare DAPI or in 1 uM DAPI solution (Life Technologies) for 15 min at RT, followed by mounting. Images were captured using B-2A and UV-2A Nikon filters, with the microscope described above.

\section{Biomechanical characterization}

The mechanical integrity of the explanted grafts was assessed under uniaxial tension. Longitudinal samples ( $n=6$ for each group) measuring $5 \mathrm{~mm}$ in length by $2.5 \mathrm{~mm}$ in width were isolated from the aortas and subjected to low strain-rate uniaxial tensile loading to failure, according to the method reported previously (Korossis et al., 2002), with minor modifications. The testing was conducted in a Zwick/Roell tensile tester (model Z 0.5) equipped with a 200N load cell. Prior to testing, the thickness of the samples was measured using a Sylvac position sensor equipped with a Kalibriert force sensor (model: $\mu$ S246). The test sample was positioned between the position sensor and a bottom plate, and the position sensor was lowered in a gradual and controllable manner, till it touched the sample and a force was registered. The distance between the position sensor and bottom plate was recorded as the sample thickness. The thickness of the samples was measured at three different points along their length, and averaged. Subsequently, the samples were clamped at their ends, using sandpaper and a small amount of superglue to prevent slippage, under zero strain on the tensile tester, which was set to produce a specimen preloading of $0.005 \mathrm{~N}$ before the operating program started to acquire any data. During testing, the specimens were preconditioned for 10 cycles at a rate of $10 \mathrm{~mm} / \mathrm{min}$, before they were sequentially stretched to failure at the same rate. Failure was assumed when the first decrease in load was detected during specimen extension, whereas the mode of failure observed was near middle-section necking and 248 rupture for all the samples tested. The sample extension $(\Delta \mathrm{l}$, in $\mathrm{mm})$ and corresponding generated load $(\mathrm{F}$, in Newtons) 249 that were recorded during the final loading to failure phase of the test, were converted to engineering strain $(\varepsilon)$ and 250 engineering stress ( $\sigma$, in MPa), respectively (Korossis et al., 2002). The stress-strain behavior of each sample was 251 analyzed by means of six parameters (Korossis et al., 2002), including the elastin (El-E) and collagen (Col-E) phase 
slopes, transition stress $\left(\sigma_{\text {Trans }}\right)$ and strain $\left(\varepsilon_{\text {Trans }}\right)$, ultimate tensile strength $\left(\sigma_{U T S}\right)$ and failure strain $\left(\varepsilon_{U T S}\right)$. The biomechanical parameters for each sample were calculated and averaged over the number of samples in each group.

\section{Data analysis}

The biomechanical testing results were presented as means together with their $95 \%$ confidence limits (C.I.). The data

was analyzed by one-way ANOVA, followed by calculation of the minimum significance difference (MSD). Statistical significance was determined at the 0.05 confidence level.

\section{Results}

\section{Histological analysis}

The histological results of the native, decellularized and explanted syngeneic and allogeneic grafts are illustrated in

Figure 1 and at higher magnification in Figure 2. The results indicated that there were no apparent differences between the histoarchitectures of native W and DA rats, with the aorta of both strains demonstrating a three-layered structure, comprising the tunica intima (outer layer of cells facing the lumen), tunica media, consisting of SMCs, collagen fibers and elastic fibers organized in a concentric fashion, and adventitia, mainly consisting of collagen fibers, fibroblasts and loosely-aligned elastic lamellae (Callanan et al., 2011; Allaire et al., 2012; Katsimpoulas et al., 2015). The decellularized DA rat aorta was shown to be completely void of any observable cells and cellular debris, while it maintained the general native trilaminar histoarchitecture with preserved collagen and elastic fibers. However, it also appeared swollen with obvious gaps in the extracellular matrix (ECM), especially at the sites in the adventitial layer that were heavily populated with cells.

The explanted syngeneic grafts (W-W; Figure $1 \& 2$ ) demonstrated an intact media with intact collagen and elastic fibers, while their adventitia appeared more compact compared to their non-implanted control (W), suggesting the production of

272 ECM. The syngeneic grafts also presented a noticeable intimal thickening and cellular content throughout their thickness. The explanted decellularized allografts (decell DA-W; Figure $1 \&$ 2) showed a similar response to syngrafts, with preserved elastin and collagen fibers in the media and a more compact adventitia compared to their non-implanted control (decell DA). Moreover, they did not show any thickening of their intima, while they demonstrated a significant cell repopulation throughout their thickness, as evidenced by the H\&E staining. The latter observation highlighted the noncytotoxic nature of the decellularised grafts, which resulted in cell colonization and subsequent ECM remodeling. On the 278 other hand, the explanted allografts (DA-W; Figure 1\& 2) demonstrated disruption of the elastin and collagen network, 
occupied by cells. Moreover, the DA-W allografts experienced cellular infiltration in the adventitia and adventitial fibrosis that resulted in a significant thickening of their adventitia, as well as neointimal formation.

\section{Immunohistochemical analysis}

283 The immunohistochemical results of the native and explanted syngeneic and allogeneic grafts are illustrated in Figure 3 284 (CD31), Figure 4 (CD68), Figure 5 (CD3 and CD4), Figure 6 (calponin), Figure 7 (CCR7) and Figure 8 (CD206). ECs $285(\mathrm{CD} 31+)$ were observed in the lumen and vasa vasorum of the native DA and W aortas, and explanted syngrafts (W-W) and untreated allografts (DA-W) (Figure 3). In contrast, no CD31+ cells were not visible on the explanted decellularized allografts (decell DA-W; Figure 3). Putative SMCs or myofibroblasts (calponin+) were observed in the tunica media of the native DA and W aortas, and the explanted syngrafts (W-W), but not in the tunica media of the explanted untreated (DAW) allografts (Figure 6). Instead, the untreated (DA-W) allografts demonstrated calponin+ cells only in their intima. No calponin+ cells were observed in the decellularized (decell DA-W) allografts (Figure 6). The native DA and W aortas were stained negative for CD68 (circulating and tissue macrophages and monocytes; Figure 4), whereas some CD68+ infiltrates were observed in the adventitia and intima of the explanted syngrafts (W-W; Figure 4). Extensive infiltration of CD68+ cells was observed in the case of the untreated allografts (DA-W), which was spread uniformly throughout the thickness of these grafts (Figure 4). CD68+ cells were also observed in the sub-endothelial region of the intima, as well as the adventitia, of the explanted decellularized allografts (decell DA-W; Figure 4). However, the infiltration of CD68+ cells in the explanted decellularized allografts was only regional, and not spread throughout the graft, as in case of the explanted untreated allografts. In spite of the lack for CD68+ staining in the native DA and W aortas, CCR7+ cells (M1 macrophage sub-type) were detected in these tissues (Figure 7). CCR7+ cells were also detected in the intima and media of the explanted syngrafts (W-W; Figure 7). Similar observations were apparent in the case of the explanted allografts (DA-W; Figure 7), in which CCR7+ infiltrates were observed in their intima and adventitia. On the other hand, the explanted decellularized allografts (decell DA-W; Figure 7).demonstrated a milder infiltration of CCR7+ cells, compared to the W-W and DA-W grafts, which was limited in their adventitia layer. No CD206+ cell infiltration was detected in any of the in native tissues (DA and W), or syngraft (W-W), untreated allograft (DA-W) or decellularised allograft (decell DA-W) explants (Figure 8).

No T-cell infiltration could be observed in the explanted syngrafts (W-W; Figure 5), whereas a significant infiltration of CD3+ cells could be observed in the adventitia of the explanted untreated allografts (DA-W). Some localised CD3+ cell presence could also be observed in the adventitia of the explanted decellularised allografts (decell DA-W; Figure 5), but 
it was considerably reduced compared to the explanted untreated allografts (DA-W). The latter also demonstrated a sporadic infiltration of CD4+ cells in their adventitia, in contrast to the explanted decellularised allografts (decell DA-W), which did show any evidence of CD4+ cell infiltration (Figure 5). In general, the explanted untreated allografts (DA-W) demonstrated a prominent infiltration of mononuclear cells, which was significantly reduced in the case of the explanted syngrafts (W-W) and decellularised allografts (decell DA-W) (Figure 4 and 5). There was no unspecific staining observed for both the secondary antibody and the isotype controls of all the above-mentioned antibody-stains (Figures 3, 4, 5, 6 , $7,8)$.

\section{Mechanical testing}

The mean biomechanical parameters of the explanted syngeneic and allogeneic grafts are illustrated in Figure 9. The same figure also features the corresponding mean biomechanical parameters of native $W(W)$ and DA (DA) rat aortas, as well as of decellularised DA rat aortas (dec DA) that were produced using the same decellularisation protocol. This data, which was obtained using the same uniaxial tensile testing protocol as in the present study, was adopted from Katsimpoulas et al. (2015) and it was included in the analysis in order to better understand the potential changes in the biomechanics of the scaffolds following implantation. Statistically significant differences were found only in the collagen phase slope (Coll-E), ultimate tensile strength ( $\left.\sigma_{U T S}\right)$ and thickness ( $p=0.025,0.027$ and 0.004 , respectively). In all three cases, the statistically significant differences arose from the differences between the explanted untreated allograft (DAW) group and the other groups tested. Specifically, the DA-W group demonstrated the lowest Coll-E compared to the other groups, with the difference being statistically significant only compared to the decellularized DA (decell DA) group (MSD $=7.22)$. Similarly, the DA-W group achieved the lowest $\sigma_{U T S}$, with the difference being statistically significant when compared to the $\mathrm{W}$ and decell DA groups (MSD $=2.07$ ). Moreover, the average thickness of the DA-W group was higher than any of the other groups, with the difference being statistically significant when compared to the native W and DA, and decell DA groups (MSD = 0.11). The explanted decellularised allograft (decell DA-W) group showed no significant differences compared to any of the other groups used in the study.

\section{Discussion}

The present study was a part of an overarching project that aimed at creating a small-caliber arterial graft for clinical use, 
animal in a future study. The orthotopic transplantation was chosen to assess the graft in the more demanding pressure

environment of the aorta. In this study, native and decellularised aortas from inbred DA rats were orthotopically implanted into $\mathrm{W}$ inbred rats to simulate the allogeneic transplantation model, whereas $\mathrm{W}$ rat native aortas were orthotopically implanted into W rats as syngeneic controls. To date, there has been a scarcity of studies on DA rat tissues implanted into W rats; nevertheless, several studies have used tissues from other sub strains of W rats for transplantation into DA rats (Mennander et al., 1991; Mennander et al., 1993). These rat strains differ in their major and minor histocompatibility loci, and the immune response in allogeneic models with these strains, are mainly driven by the MHC I and II present on donor cells (Schmitz-Rixen et al., 1988). In this study, lymphocytes presence was investigated using anti-CD3 and anti-CD4 antibodies, since CD3 has been reported to be a pan-T-cell marker (Aniansson Zdolsek et al., 1999) and CD4 a marker for T-regulatory and T-helper cells (Corthay et al., 2009). Macrophages were detected by the anti-CD68 antibody, which has been reported as a marker for pan-macrophages (Murray et al., 2011), whereas the M1 and M2 macrophage sub-types were detected by anti-CCR7 and anti-CD206 immunofluorescent antibodies, respectively. M1 macrophages are typically activated by IFN-y or lipopolysaccharides, producing proinflammatory cytokines, and are indicative of an immune response, whereas M2 macrophages are activated by cytokines, such as IL-

4, IL-10, or IL-13, and produce either proliferation-inducing polyamines or proline to induce collagen production. M2 macrophages have been associated with wound healing and tissue repair and remodeling (Brown et al.,2009; Valentin et al., 2009; Brown et al., 2012; Jablonski et al., 2015; Sager et al., 2017)

Allograft transplantation usually leads to chronic rejection, which mainly consists of inflammation and intimal thickening (Mennander et al., 1992). In particular, this degenerative process induces a complete loss of SMCs in the media that, in turn, induces media necrosis, elastin degradation, SMC migration towards the intima and intimal thickening throughout the length of the graft (Mennander et al., 1993). It has been hypothesized that medial necrosis might be due to a toxic effect of inflammatory cells present in the adventitia, especially lymphocytes, on SMCs in the media (Mennander et al., 1993). In the present study, media necrosis, partial elastic fiber degradation, SMC migration towards the intima and lymphocyte $(\mathrm{CD} 3+, \mathrm{CD4}+)$ and macrophage $(\mathrm{CD68}+)$ infiltration in the adventitia were evidenced in the explanted untreated allografts (DA-W), which also demonstrated a prominent increase in thickness of their adventitia (Figure 1 and 2). Donor ECs and SMCs were most probably the main antigenic targets of these infiltrates, since the DA-W grafts were stained positively for CD31 in their lumen (Figure 3) and presented a significant content of calponin+ cells in their intima 
Furth rats. The authors reported a peak of inflammatory cells and increase in adventitial thickness after 2 months implantation.

The syngrafts showed a mild immune response, with an intact media (Figure 2) and no lymphocyte presence (Figure 5). Macrophages were observed in all explants, located mainly in the intima, media and adventitia of the allografts, and in the media and intima of the decellularized allografts and syngrafts (Figure 4). In all explants, the macrophages were identified as of the M1 sub-type (inflammatory; Figure 7), whereas no M2 macrophages (remodeling/repair) were observed in any of the explants (Figure 8). In contrast to the DA-W allografts, the explanted decellularized allografts (decell DA-W) induced an immune response similar to the syngrafts (W-W), with a reduced immune injury (Figure 2), low amount of lymphocyte infiltrates (Figure 5), and macrophages that were localized mainly in regions of the adventitia (Figure 7), although some macrophages were also observed in their intima region, as evidenced under CD68 staining (Figure 4). This regional macrophage presence in the decell DA-W allografts might be due to inconsistencies in the counterparts, and that the decellularisation protocol was generally effective in removing the immunogenic material, such as cellular debris, of the grafts.

The decellularized aortas, both prior (decell DA) and after (decell DA-W) transplantation, showed a well maintained trilaminar structure, typical to abdominal rat aorta (Allaire et al., 1994), with well-maintained collagen and elastic fibers orientated concentrically along the circumferential direction in the media, and elastin lamellae in the adventitia (Figure 1 and 2). Elastic fibers and lamellae represent one of the main components of the aortic wall (Aikawa et al., 2009; Cavalcante et al., 2011). Reduction in the number of elastic fibers and elastic lamellae has been reported to cause a reduction of mural elastic resistance (Mello et al., 2004) that could contribute to aneurysm formation (Boutouyrie et al., 1992). The histological analysis of the decellularized aortas prior to implantation revealed absence of any observable cells or cellular debris throughout the thickness of the arterial wall. The explanted decell DA-W allografts demonstrated extensive cellular population in their media and adventitia, as well as evidence of remodeling of the adventitia, which was rather disrupted by the decellularisation process (Figure 2). However, no ECs could be observed in the lumen of the decell DA-W allografts, as evidenced by the CD31 staining (Figure 3), or any detectable SMC presence, as evidenced by 
adventitia, probably due to the recruitment of fibroblasts by inflammatory cells, in an attempt to replace the allogeneic tissue (Mennander et al., 1992), as well as neointima formation (Figure 2).

The histoarchitectural changes in the explanted DA-W allografts (Figure 1 and 2) had a direct effect on their mechanical properties. Specifically, the DA-W group demonstrated on average the lowest Coll-E and $\sigma_{\text {UTS, }}$ and the highest macroscopic thickness, among the groups tested, indicating a deterioration of the integrity of these grafts (Figure 9). These changes in the mechanical properties of the DA-W allografts have the potential to induce long-term structural failure. Moreover, the reduced moduli measured for the DA-W explants, implied that there was a significant modulus mismatch between these allografts and the native aorta of the recipients. This could have potentially generated significant stress concentrations in the grafts and/or the surrounding aorta of the recipient, generating abnormal biomechanical stimuli to infiltrating cells. In addition, the high stress concentration regions could have potentially generated higher ECM damage, further attracting inflammatory and immune cells, and increasing the immunogenicity of these grafts. On the other hand, and in the absence of a functional vasa vasorum, the increase in the DA-W graft thickness in vivo could have generated progressively increased hypoxic conditions in the media of these grafts, which in turn caused higher migration of immune cells, such as macrophages, macrophages, neutrophils, dendritic cells, lymphocytes and immune lymphoid cells, whose characteristic ability is to infiltrate in tissues with low nutrients and oxygen levels (Krzywinska et al., 2018).

In contrast, the explanted decell DA-W allografts showed no significant differences in the mechanical properties compared to the native W rat aorta. Overall, these results suggested that the ECM of explanted decellularized scaffolds was more stable and better maintained after 6 weeks implantation, in contrast to that of the untreated DA-W allografts, which showed partial disruption of their elastic fibres, adventitia thickening and deteriorated mechanical properties, caused by the immune response of recipients.

In conclusion, the results from this study demonstrated that the modified decellularisation protocol used in the present study did not induce significant biomechanical or histological alterations of the DA rat aorta in vivo, whereas the immune response of the recipients was improved by the decellularisation treatment compared to the untreated allografts. The latter elicited a significant adverse immune response, which resulted in adventitia fibrosis and thickening, media necrosis and neointima formation, whereas the syngrafts showed good tissue integration and mild immune response. Future work will assess the presence of nucleic acid and phospholipid remnants in the decellularised scaffolds. Moreover, future work 
will also assess the performance, remodeling and adaptation of the decellularised rat aorta graft in a heterotopic peripheral artery porcine model.

\section{Acknowledgements}

421 The authors would like to acknowledge the contribution of Karin Burgwitz at the Lower Saxony Centre for Biomedical 422 Engineering Implant Research and Development for their technical support. This research was supported by the People 423 Programme (Marie Curie Actions) of the EU 7 Framework Programme FP7/2007-2013/ under the REA grant agreement 424 number 317512, and the German Research Foundation through the Cluster of Excellence REBIRTH (From 425 Regenerative Biology to Reconstructive Therapy; EXC 62).

\section{Author Disclosure Statement}

No competing financial interests exist for all authors.

\section{References}

Affonso da Costa FDP, Dohmen M, Duarte D, von Glenn C, Veiga Lopes S, Filho HH, Affonso da Costa MB, Konertz W. Immunological and echocardiographic evaluation of decellularized versus cryopreserved allografts during the Ross operation, European Journal of Cardio-Thoracic Surgery, Volume 27, Issue 4, 1 April 2005, Pages 572-578.

Aikawa E, Aikawa M, Rusanescu G, Iwamoto Y, Shi GP, Jaffer FA et al. Arterial and aortic valve calcification abolished by elastolytic cathepsin S deficiency in chronic renal disease. Circulation 2009; 119, 1785-1794.

Allaire E, Guettier C, Bruneval P, Plissonnier D, Michel JB Cell-free arterial grafts: morphologic characteristics of aortic isografts, allografts, and xenografts in rats. Journal of Vascular Surgery: Official Publication, the Society for Vascular Surgery [and] International Society for Cardiovascular Surgery, North American Chapter 1994; 19, 446-456.Guillen J. FELASA guidelines and recommendations. J Am Assoc Lab Anim Sci 2012; 51(3):311-21.

438 Aniansson Zdolsek H, Ernerudh J, Holt PG, Nilsson J, Björkstén B. Expression of the T-cell markers CD3, CD4 and CD8 439 in healthy and atopic children during the first 18 months of life. Int Arch Allergy Immunol. 1999 May; 119(1):6-12.

440 Aper T, Wilhelmi M, Gebhardt C, Hoeffler K, Benecke N, Hilfiker A, Haverich A. Novel method for the generation of 441 tissue-engineered vascular grafts based on a highly compacted fibrin matrix. Acta Biomaterialia, 2016;29:21-32.

442 Atala, A., et al., Tissue-engineered autologous bladders for patients needing cystoplasty. Lancet, 2006. 367(9518): $p$. $443 \quad 1241-6$. 
Bobylev, D., et al., Semilunar valve replacement with decellularized homograft after Damus-Kaye-Stansel anastomosis and fontan procedure. Ann Thorac Surg, 2014. 97(5): p. 1792-5.

Boutouyrie P, Laurent S, Benetos A, Girerd XJ, Hoeks AP, Safar ME Opposing effects of ageing on distal and proximal large arteries in hypertensives. Journal of Hypertension. Supplement: Official Journal of the International Society of Hypertension 1992; 10, S87-S91.

Brown BN, Valentin JE, Stewart-Akers AM, McCabe GP, Badylak SF. Macrophage phenotype and remodeling outcomes in response to biologic scaffolds with and without a cellular component. Biomaterials. 2009 Mar;30(8):1482-91. as a predictor of constructive remodeling following the implantation of biologically derived surgical mesh materials. Acta Biomater. 2012 Mar;8(3):978-87.

Callanan A, Davis NF, Walsh MT, McGloughlin TM Tissue-Engineered Extracellular Matrices (ECMs) as Adjuvant Scaffolds for Endovascular Aneurysmal Repair (EVAR). In Regenerative Medicine and Tissue Engineering - Cells and Biomaterials 2011; 301-322.

Cavalcante JL, Lima JAC, Redheuil A, Al-Mallah MH Aortic stiffness: Current understanding and future directions. Journal of the American College of Cardiology 2011.

Chaouat M, Le Visage C, Autissier A, Chaubet F, Letourneur D. The evaluation of a small-diameter polysaccharidebased arterial graft in rats. Biomaterials. 2006; 27(32):5546-5553.

Clark RA, Nielsen LD, Welch MP, McPherson JM. Collagen matrices attenuate the collagen-synthetic response of cultured fibroblasts to TGF-beta. J Cell Sci. 1995;108(Pt 3):1251-61.

Corthay A. How do Regulatory T Cells Work? Scandinavian Journal of Immunology. 2009; 70(4):326-336.

Criqui $\mathrm{MH}$, Fronek $\mathrm{A}$, Barrett-Connor $\mathrm{E}$, et al. The prevalence of peripheral arterial disease in a defined population. Circulation 1985; 71:510-515. 685426. acellular Matrix. Annals of Plastic Surgery 2009; 62(3). 
Dietrich M, Heselhaus J, Wozniak J, Weinandy S, Mela P, Tschoeke B, Schmitz-Rode T, and Jockenhoevel S. FibrinBased Tissue Engineering: Comparison of Different Methods of Autologous Fibrinogen Isolation Tissue Engineering Part C: Methods. December 2012, 19(3): 216-226.

Fowkes FG, Housley E, Cawood EH, et al. Edinburgh artery study: prevalence of asymptomatic and symptomatic peripheral arterial disease in the general population. Int J Epidemiol. 1991; 20:384-392.

Gui, L., et al., Development of decellularized human umbilical arteries as small diameter vascular grafts. Tissue Eng Part A, 2009. 15(9): p. 2665-76.

Gui L, et al. Novel utilization of serum in tissue decellularisation. Tissue Eng Part C Methods 2010; 16(2):173-84.

Hashimoto, Y., et al. Preparation and characterization of decellularized cornea using high-hydrostatic pressurization for corneal tissue engineering. Biomaterials, 2010. 31(14): p. 3941-8.

Hwang, S.J., et al., The decellularized vascular allograft as an experimental platform for developing a biocompatible small-diameter graft conduit in a rat surgical model. Yonsei Med J, 2011. 52(2): p. 227-33.

Ingram, J.H., et al. The use of ultrasonication to aid recellularization of acellular natural tissue scaffolds for use in anterior cruciate ligament reconstruction. Tissue Eng, 2007. 13(7): p. 1561-72.

Jablonski KA, Amici SA, Webb LM, et al. Novel Markers to Delineate Murine M1 and M2 Macrophages. Olszewski MA, ed. PLoS ONE. 2015;10(12):e0145342. doi:10.1371/journal.pone.0145342.

Kakisis JD, Liapis CD, Breuer C, et al. Artificial blood vessel: the Holy Grail of peripheral vascular surgery. J. Vasc. Surg. $2005 ; 41: 349-54$.

Katsimpoulas M, Morticelli L, et al. Investigation of the biomechanical integrity of decellularized rat abdominal aorta. Transplant Proc. 2015 May;47(4):1228-33.

Korossis SA, Booth C, Wilcox HE, Watterson KG, Kearney JN, Fisher J, et al. Tissue engineering of cardiac valve prostheses II: biomechanical characterization of decellularized porcine aortic heart valves. The Journal of Heart Valve Disease 2002; 11, 463-471.

Koyama, S., et al. Optimal bypass graft design for left anterior descending and diagonal territory in multivessel coronary disease. Interact Cardiovasc Thorac Surg, 2014. 19(3): p. 406-13. 
Krzywinska E, Stockmann C. Hypoxia, Metabolism and Immune Cell Function. Biomedicines. 2018;6(2):56.

496

497

498

499

500

501

502

503

504

doi:10.3390/biomedicines6020056.

Luo J, Korossis SA, Wilshaw SP, Jennings LM, Fisher J, Ingham E. Development and characterization of acellular porcine pulmonary valve scaffolds for tissue engineering. Tissue Eng Part A. 2014 Nov;20(21-22):2963-74.

Mello JM De, Orsi AM, Padovani CR, Maria S, Matheus M, Eleutério ML Structure of the aortic wall in the guinea pig and rat $2004 ; 21,35-38$.

Mennander A, Tiisala S, Halttunen J, Yilmaz S, Paavonen T, Häyry P. Chronic Rejection in Rat Aortic Allografts An Experimental Model for Transplant Arteriosclerosis. Arteriosclerosis and Thrombosis 1991; Il:671-680.

Mennander A, Tiisala S, Ustinov J, Raisanen A, Paavonen T, Häyry P. Chronic rejection of rat aortic allografts: synthesis of the major eicosanoids by the vascular wall components, and the effect of inhibition of the thromboxane cascade. Arterioscler Thromb 1992;12:1380-1386.

Mennander A, Paavonen T and Häyry P. Intimal Thickening and Medial Necrosis in Allograft Arteriosclerosis (Chronic Rejection) Are Independently Regulated. Arteriosclerosis and Thrombosis 1993; 13:1019-102.

Murray PJ, Wynn TA. Protective and pathogenic functions of macrophage subsets. Nat Rev Immunol. 2011 Oct 14; 11(11):723-37.

Neumann A, Sarikouch S, Breymann T, Cebotari S, Boethig D, Horke A, Beerbaum P, Westhoff-Bleck M, Bertram H, Ono M, Tudorache I, Haverich A, Beutel G. Early systemic cellular immune response in children and young adults receiving decellularized fresh allografts for pulmonary valve replacement. Tissue Eng Part A. 2014 Mar; 20(5-6):1003-11.

Onuta G, Rienstra H, de Boer JF, Boer MW, Roks AJ, Klatter FA, Uges DR, Navis G, Rozing J, Hillebrands JL. Rosiglitazone attenuates transplant arteriosclerosis after allogeneic aorta transplantation in rats. Transplantation. 2007; 84(4):517-26.

Paniagua GJR., Berry H, Korossis S, Mirsadraee S, Lopes SV, Da Costa F, Kearney J, Watterson K, Fisher J, Ingham E. Regenerative potential of low-concentration SDS-decellularized porcine aortic valved conduits in vivo. Tissue Engineering - Part A. 2015; 21 (1-2), pp. 332-342.

Roh JD, Sawh-Martinez R, Brennan MP, Jay SM, Devine L, Rao DA, Yi T, Mirensky TL, Nalbandian A, Udelsman B, Hibino N, Shinoka T, Saltzman WM, Snyder E, Kyriakides TR, Pober JS, Breuer CK. Tissue-engineered vascular grafts 
transform into mature blood vessels via an inflammation-mediated process of vascular remodeling. Proceedings of the National Academy of Sciences of the United States of America. 2010;107(10):4669-4674.

Sager HB, Kessler T, Schunkert H. Monocytes and macrophages in cardiac injury and repair. Journal of Thoracic Disease. 2017;9(Suppl 1):S30-S35.

Santoso, E.G., et al., Application of detergents or high hydrostatic pressure as decellularisation processes in uterine tissues and their subsequent effects on in vivo uterine regeneration in murine models. PLoS One, 2014. 9(7): $p$. e103201.

Sarikouch S, Horke A, Tudorache I, Beerbaum P, Westhoff-Bleck M, Boethig D, Repin O, Maniuc L, Ciubotaru A, Haverich A, Cebotari S. Decellularized fresh homografts for pulmonary valve replacement: a decade of clinical experience. European Journal of Cardio-Thoracic Surgery, Volume 50, Issue 2, 1 August 2016, Pages 281-290.

Schmitz-Rixen T, Megerman J, Colvin RB, Williams AM, Abbott WM. Immunosuppressive treatment of aortic allografts. J VAsc SURG 1988; 7:82-92.

Schneider-Bartholda C, Baganza S, Wilhelmi M, Scheper T and Pepelanova I. Hydrogels based on collagen and fibrin frontiers and applications. BioNanoMat 2016; 17(1-2): 3-12.

Sokal R, Rohlf F Single classification analysis of variance, in: Biometry. W.H. Freeman \& Co. 1981; 208-270.

Struecker, B., et al., Porcine liver decellularisation under oscillating pressure conditions - A technical refinement to improve the homogeneity of the decellularisation process. Tissue Eng Part C Methods, 2014.

Swartz DD, Russell JA, Andreadis ST. Engineering of fibrin-based functional and implantable small-diameter blood vessels. American Journal of Physiology-Heart and Circulatory Physiology. 2005;288(3):H1451-H1460.

Valentin JE, Stewart-Akers AM, Gilbert TW, Badylak SF. Macrophage Participation in the Degradation and Remodeling of Extracellular Matrix Scaffolds. Tissue Engineering Part A. 2009;15(7):1687-1694.

Whittermore $A D$, Kent $K C$, Donaldson $M C$, et al. What is the proper role of polytetrafluoroethylene grafts in infrainguinal reconstruction? J. Vasc. Surg. 1989; 10:299-305.

Yu, W., J.R. Wright, Jr., and J.J. Appoo. Venous intimal hyperplasia with occlusion of the anastomosis between saphenous vein graft and carbo-seal dacron tube after a modified Bentall procedure. J Heart Valve Dis, 2013. 22(6): $p$. $867-71$. 
547 Zhang WJ, Liu W, Cui L, Cao Y. Tissue engineering of blood vessel. J Cell Mol Med. 2007; 11(5):945-957.

548 Zorlutuna P, Elsheikh A, Hasirci V. Nanopatterning of collagen scaffolds improve the mechanical properties of tissue 549 engineered vascular grafts. Biomacromolecules. 2009; 10(4):814-821. 